\title{
Chapter 34 \\ Maximal Differentiation in the Hotelling Model with Uncertainty
}

\author{
Alberto Adrego Pinto and Telmo Parreira
}

\subsection{Introduction}

Since the seminal work of Hotelling [14], the model of spatial competition has been seen by many researchers as an attractive framework for analyzing oligopoly markets (see [2,13,15-21,24,25]). In his model, Hotelling present a city represented by a line segment where a uniformly distributed continuum of consumers have to buy a homogeneous good. Consumers have to support linear transportation costs when buying the good in one of the two firms of the city. The firms compete in a two-staged location-price game, where simultaneously choose their location and afterwards set their prices in order to maximize their profits. Hotelling concluded that firms would agglomerate at the center of the line, an observation referred as the "Principle of Minimum Differentiation".

In 1979, D'Aspremont et al. [4] show that the "Principle of Minimum Differentiation" is invalid, since there was no price equilibrium solution for all possible locations of the firms, in particular when they are not far enough from each other. Moreover, in the same article, D'Aspremont et al. introduce a modification in the Hotelling model, considering quadratic transportation costs instead of linear. The introduction of this feature removed the discontinuities verified in the profit and

\footnotetext{
A.A. Pinto

LIAAD-INESC TEC, Porto, Portugal

Faculty of Sciences, Department of Mathematics, University of Porto, Rua do Campo Alegre, 687, 4169-007, Porto, Portugal

e-mail: aapinto@fc.up.pt

T. Parreira $(\bowtie)$

Department of Mathematics, University of Minho, Campus de Gualtar, 4710-057 Braga, Portugal

LIAAD-INESC TEC, Porto, Portugal

e-mail: telmoparreira@hotmail.com
} 
demand functions, which was a problem in Hotelling model and they show that, under quadratic transportation costs, a price equilibrium exists for all locations and a location equilibrium exists and involves maximum product differentiation, i.e. the firms opt to locate at the extremes of the line.

Hotelling and D'Aspremont et al. consider that the production costs of both firms are equal to zero. Ziss [26] introduce a modification in the model of D'Aspremont et al. by allowing for different production costs between the two firms and examines the effect of heterogeneous production technologies on the location problem. Ziss shows that a price equilibrium exists for all locations and concludes that when the difference between the production costs is small, a price and location equilibrium exists in which the firms prefer to locate in different extremes of the line. However, if the difference between the production costs is sufficiently large, a location equilibrium does not exist.

Boyer et al. [3] and Biscaia and Sarmento [1] extended the work of Ziss by consider that the uncertainty on the productions costs exists only during the first subgame in location strategies. Then the production costs are revealed to the firms before the firms have to choose their optimal price strategies and so the second subgame has complete information.

In this work, we study the quadratic Hotelling model with incomplete information in the production costs of both firms. The incomplete information consists in each firm to know its production cost but to be uncertain about the competitor cost as usual in oligopoly theory (see [5-12,22,23]). However, in contrast with Boyer et al. [3], the production costs are not revealed to the firms before the firms have to choose their price strategy. Furthermore, our results are universal, in the incomplete information scenario, in the sense that they apply to all probability distributions in the production costs.

We say that the Bayesian-Nash price strategy has the duopoly property if both firms have non-empty market for every pair of production costs. We introduce the bounded uncertain costs and location BUCL1 condition that defines a bound for the production costs in terms only of the exogenous variables that are the transportation cost; the road length of the segment line; and the localization of both firms (see Sect. 34.6). We prove that there is a local optimum price strategy with the duopoly property if and only if the bounded uncertain costs and location BUCL1 condition holds. We compute explicitly the formula for the local optimum price strategy that is simple and leaves clear the influence of the relevance economic exogenous quantities in the price formation. In particular, we observe that the local optimum price strategy do not depend on the distributions of the production costs of the firms, except on their first moments.

We introduce two mild additional bounded uncertain costs and location $B U C L 2$ and $B U C L 3$ conditions. Under the BUCL1 and BUCL2 conditions, we prove that the local optimum price strategy is a Bayesian-Nash price strategy. Assuming that the firms choose the Bayesian-Nash price strategy, under the BUCL3 condition, we prove that the maximal differentiation is a local optimum for the localization strategy of both firms.

All the results presented in this chapter are proved in [21]. 


\subsection{Local Optimum Price Strategy Under Complete Information}

The buyers of a commodity will be supposed uniformly distributed along a line with length $l$, where two firms $A$ and $B$ located at respective distances $a$ and $b$ from the endpoints of the line sell the same commodity with unitary production $\operatorname{costs} c_{A}$ and $c_{B}$. We assume without loss of generality that $a \geqslant 0, b \geqslant 0$ and $l-a-b \geqslant 0$. No customer has any preference for either seller except on the ground of price plus transportation cost $t$. We will assume that each consumer buys a single unit of the commodity, in each unit of time and in each unit of length of the line. Denote A's price by $p_{A}$ and B's price by $p_{B}$. The point of division $\left.x=x\left(p_{A}, p_{B}\right) \in\right] 0, l[$ between the regions served by the two entrepreneurs is determined by the condition that at this place it is a matter of indifference whether one buys from $A$ or from $B$. The point $x$ is the location of the indifferent consumer to buy from firm $A$ or firm $B$, if

$$
p_{A}+t(x-a)^{2}=p_{B}+t(l-b-x)^{2}
$$

Let

$$
m=l-a-b ; \quad \Delta_{l}=a-b \quad \text { and } \quad \Delta_{C}=c_{A}-c_{B} .
$$

Solving for $x$, we obtain

$$
x=\frac{p_{B}-p_{A}}{2 t m}+\frac{l+\Delta_{l}}{2} .
$$

Both firms have a non-empty market share if, and only if, $x \in] 0, l[$. Hence, the prices will have to satisfy

$$
\left|p_{A}-p_{B}-t m \Delta_{l}\right|<t m l
$$

Assuming inequality (34.1), both firms $A$ and $B$ have a non-empty demand ( $x$ and $l-x)$ and the profits of the two firms are defined respectively by

$$
\pi_{A}=\left(p_{A}-c_{A}\right) x=\left(p_{A}-c_{A}\right)\left(\frac{p_{B}-p_{A}}{2 t m}+\frac{l+\Delta_{l}}{2}\right)
$$

and

$$
\pi_{B}=\left(p_{B}-c_{B}\right)(l-x)=\left(p_{B}-c_{B}\right)\left(\frac{p_{A}-p_{B}}{2 t m}+\frac{l-\Delta_{l}}{2}\right) .
$$

Definition 34.1. A price strategy $\left(p_{A}, p_{B}\right)$ for both firms is a local optimum price strategy if (i) for every small deviation of the price $p_{A}$ the profit $\pi_{A}$ of firm $A$ 
decreases, and for every small deviation of the price $p_{B}$ the profit $\pi_{B}$ of firm $B$ decreases (local optimum property); and (ii) the indifferent consumer exists, i.e. $0<x<l$ (duopoly property).

Let us compute the local optimum price strategy $\left(p_{A}, p_{B}\right)$. Differentiating $\pi_{A}$ with respect to $p_{A}$ and $\pi_{B}$ with respect to $p_{B}$ and equalizing to zero, we obtain the first order conditions (FOC). The FOC implies that

$$
p_{A}=t m\left(l+\frac{\Delta_{l}}{3}\right)+c_{A}-\frac{\Delta_{C}}{3}
$$

and

$$
p_{B}=t m\left(l-\frac{\Delta_{l}}{3}\right)+c_{B}+\frac{\Delta_{C}}{3} .
$$

We note that the first order conditions refer to jointly optimizing the profit function (34.2) with respect to the price $p_{A}$ and the profit function (34.3) with respect to the price $p_{B}$.

Since the profit functions (34.2) and (34.3) are concave, the second-order conditions for this maximization problem are satisfied and so the prices (34.4) and (34.5) are indeed maxima for the functions (34.2) and (34.3), respectively. The corresponding equilibrium profits are given by

$$
\pi_{A}=\frac{\left(m\left(3 l+\Delta_{l}\right) t-\Delta_{C}\right)^{2}}{18 t m}
$$

and

$$
\pi_{B}=\frac{\left(m\left(3 l-\Delta_{l}\right) t+\Delta_{C}\right)^{2}}{18 t m} .
$$

Furthermore, the consumer indifference location corresponding to the maximizers $p_{A}$ and $p_{B}$ of the profit functions $\pi_{A}$ and $\pi_{B}$ is

$$
x=\frac{l}{2}+\frac{\Delta_{l}}{6}-\frac{\Delta_{C}}{6 t m} .
$$

Finally, for the pair of prices $\left(p_{A}, p_{B}\right)$ to be a local optimum price strategy, we need assumption (34.1) to be satisfied with respect to these pair of prices. We observe that assumption (34.1) is satisfied with respect to the pair of prices $\left(p_{A}, p_{B}\right)$ if and only if the following condition with respect to the production costs is satisfied.

Definition 34.2. The Hotelling model satisfies the bounded costs and location $(B C L)$ condition, if

$$
\left|\Delta_{C}-t m \Delta_{l}\right|<3 t m l .
$$


We note that under the $B C L$ condition the prices are higher than the production costs $p_{A}>c_{A}$ and $p_{B}>c_{B}$. Hence, there is a local optimum price strategy if and only if the $B C L$ condition holds. Furthermore, under the $B C L$ condition, the pair of prices $\left(p_{A}, p_{B}\right)$ is the local optimum price strategy.

A strong restriction that the $B C L$ condition imposes is that $\Delta_{C}$ converges to 0 when $m$ tends to 0 , i.e. when the differentiation in the localization tends to vanish.

\subsection{Nash Price Strategy Under Complete Information}

We note that, if a Nash price equilibrium satisfies the duopoly property then it is a local optimum price strategy. However, a local optimum price strategy is only a local strategic maximum. Hence, the local optimum price strategy to be a Nash equilibrium must also be global strategic maximum. In this section, we are going to show that this is the case.

Following D'Aspremont et al. [4], we note that the profits of the two firms, valued at local optimum price strategy are globally optimal if they are at least as great as the payoffs that firms would earn by undercutting the rivals's price and supplying the whole market.

Let $\left(p_{A}, p_{B}\right)$ be the local optimum price strategy. Firm $A$ may gain the whole market, undercutting its rival by setting

$$
p_{A}^{M}=p_{B}-t m\left(l-\Delta_{l}\right) .
$$

In this case the profit amounts to

$$
\pi_{A}^{M}=\frac{2}{3}\left(t m \Delta_{l}-\Delta_{C}\right) l
$$

A similar argument is valid for store $B$. Undercutting this rival, setting

$$
p_{B}^{M}=p_{A}-t m\left(l+\Delta_{l}\right),
$$

it would earn

$$
\pi_{B}^{M}=\frac{2}{3}\left(\Delta_{C}-t m \Delta_{l}\right) l
$$

The conditions for such undercutting not to be profitable are $\pi_{A} \geqslant \pi_{A}^{M}$ and $\pi_{B} \geqslant$ $\pi_{B}^{M}$. Hence, proving that

$$
\frac{\left(m\left(3 l+\Delta_{l}\right) t-\Delta_{C}\right)^{2}}{18 t m} \geqslant \frac{2}{3}\left(t m \Delta_{l}-\Delta_{C}\right) l
$$


is sufficient to prove that $\pi_{A} \geqslant \pi_{A}^{M}$. Similarly, proving that

$$
\frac{\left(m\left(3 l-\Delta_{l}\right) t+\Delta_{C}\right)^{2}}{18 t m} \geqslant \frac{2}{3}\left(\Delta_{C}-t m \Delta_{l}\right) l
$$

is sufficient to prove that $\pi_{B} \geqslant \pi_{B}^{M}$.

However, conditions (34.7) and (34.8) are satisfied because they are equivalent to

$$
\left(m\left(3 l-\Delta_{l}\right) t+\Delta_{C}\right)^{2} \geqslant 0
$$

and

$$
\left(m\left(3 l+\Delta_{l}\right) t-\Delta_{C}\right)^{2} \geqslant 0 .
$$

Therefore, if $\left(p_{A}, p_{B}\right)$ is a local optimum price strategy then $\left(p_{A}, p_{B}\right)$ is a Nash price equilibrium.

\subsection{Optimum Localization Equilibrium Under Complete Information}

We are going to find when the maximal differentiation is a local optimum strategy assuming that the firms in second subgame choose the Nash price equilibrium strategy. For a complete discussion see Ziss [26].

We note that from (34.4) and (34.6), we can write the profit of firm $A$ as

$$
\pi_{A}=\frac{\left(p_{A}-c_{A}\right)^{2}}{2 t(l-a-b)}
$$

Since

$$
\frac{\partial p_{A}}{\partial a}=-\frac{2}{3} t(l+a)
$$

we obtain that

$$
\frac{\partial \pi_{A}}{\partial a}=-\frac{p_{A}-c_{A}}{6 t(l-a-b)^{2}}\left(\Delta_{C}+t(l-a-b)(l+3 a+b)\right) .
$$

Similarly, we obtain that

$$
\frac{\partial \pi_{B}}{\partial b}=\frac{p_{B}-c_{B}}{6 t(l-a-b)^{2}}\left(\Delta_{C}-t(l-a-b)(l+a+3 b)\right) .
$$


Therefore, the maximal differentiation $(a, b)=(0,0)$ is a local optimum strategy if and only if

$$
\frac{\partial \pi_{A}}{\partial a}(0,0)=-\frac{p_{A}-c_{A}}{6 t l^{2}}\left(\Delta_{C}+t l^{2}\right)<0
$$

and

$$
\frac{\partial \pi_{B}}{\partial b}(0,0)=\frac{p_{B}-c_{B}}{6 t l^{2}}\left(\Delta_{C}-t l^{2}\right)<0
$$

Since

$$
\frac{p_{A}-c_{A}}{6 t l^{2}}>0 \text { and } \quad \frac{p_{B}-c_{B}}{6 t l^{2}}>0
$$

the maximal differentiation $(a, b)=(0,0)$ is a local optimum strategy if and only if

$$
\left|\Delta_{C}\right|<t l^{2}
$$

\subsection{Incomplete Information on the Production Costs}

The incomplete information consists in each firm to know its production cost but to be uncertain about the competitor cost. In this section, we introduce a simple notation that is fundamental for the elegance and understanding of the results presented in this paper.

Let the triples $\left(I_{A}, \Omega_{A}, q_{A}\right)$ and $\left(I_{B}, \Omega_{B}, q_{B}\right)$ represent (finite, countable or uncountable) sets of types $I_{A}$ and $I_{B}$ with $\sigma$-algebras $\Omega_{A}$ and $\Omega_{B}$ and probability measures $q_{A}$ and $q_{B}$, over $I_{A}$ and $I_{B}$, respectively.

We define the expected values $E_{A}(f), E_{B}(f)$ and $E(f)$ with respect to the probability measures $q_{A}$ and $q_{B}$ as follows:

$$
E_{A}(f)=\int_{I_{A}} f(z, w) \mathrm{d} q_{A}(z) ; \quad E_{B}(f)=\int_{I_{B}} f(z, w) \mathrm{d} q_{B}(w)
$$

and

$$
E(f)=\int_{I_{A}} \int_{I_{B}} f(z, w) \mathrm{d} q_{B}(w) \mathrm{d} q_{A}(z)
$$

Let $c_{A}: I_{A} \rightarrow \mathbb{R}_{0}^{+}$and $c_{B}: I_{B} \rightarrow \mathbb{R}_{0}^{+}$be measurable functions where $c_{A}^{z}=c_{A}(z)$ denotes the production cost of firm $A$ when the type of firm $A$ is $z \in I_{A}$ and $c_{B}^{w}=$ $c_{B}(w)$ denotes the production cost of firm $B$ when the type of firm $B$ is $w \in I_{B}$. Furthermore, we assume that the expected values of $c_{A}$ and $c_{B}$ are finite 


$$
E\left(c_{A}\right)=E_{A}\left(c_{A}\right)=\int_{I_{A}} c_{A}^{z} \mathrm{~d} q_{A}(z)<\infty ; E\left(c_{B}\right)=E_{B}\left(c_{B}\right)=\int_{I_{B}} c_{B}^{w} \mathrm{~d} q_{B}(w)<\infty .
$$

We assume that $\mathrm{d} q_{A}(z)$ denotes the probability of the belief of the firm $B$ on the production costs of the firm $A$ to be $c_{A}^{z}$. Similarly, assume that $\mathrm{d} q_{B}(w)$ denotes the probability of the belief of the firm $A$ on the production costs of the firm $B$ to be $c_{B}^{w}$.

The simplicity of the following cost deviation formulas is crucial to express the main results of this article in a clear and understandable way. The cost deviations of firm $A$ and firm $B$

$$
\Delta_{A}: I_{A} \rightarrow \mathbb{R}_{0}^{+} \quad \text { and } \quad \Delta_{B}: I_{B} \rightarrow \mathbb{R}_{0}^{+}
$$

are given respectively by $\Delta_{A}(z)=c_{A}^{z}-E\left(c_{A}\right)$ and $\Delta_{B}(w)=c_{B}^{w}-E\left(c_{B}\right)$. The cost deviation between the firms

$$
\Delta_{C}: I_{A} \times I_{B} \rightarrow \mathbb{R}_{0}^{+}
$$

is given by $\Delta_{C}(z, w)=c_{A}^{z}-c_{B}^{w}$. Since the meaning is clear, we will use through the paper the following simplified notation:

$$
\Delta_{A}=\Delta_{A}(z) ; \quad \Delta_{B}=\Delta_{B}(w) \text { and } \Delta_{C}=\Delta_{C}(z, w) .
$$

The expected cost deviation $\Delta_{E}$ between the firms is given by $\Delta_{E}=E\left(c_{A}\right)-E\left(c_{B}\right)$. Hence,

$$
\Delta_{C}-\Delta_{E}=\Delta_{A}-\Delta_{B}
$$

\subsection{Local Optimum Price Strategy Under Complete Information}

In this section, we introduce incomplete information in the classical Hotelling game and we find the local optimal price strategy. We introduce the bounded uncertain costs condition that allows us to find the local optimum price strategy.

A price strategy $\left(p_{A}, p_{B}\right)$ is given by a pair of functions $p_{A}: I_{A} \rightarrow \mathbb{R}_{0}^{+}$and $p_{B}: I_{B} \rightarrow \mathbb{R}_{0}^{+}$where $p_{A}^{z}=p_{A}(z)$ denotes the price of firm $A$ when the type of firm $A$ is $z \in I_{A}$ and $p_{B}^{w}=p_{B}(w)$ denotes the price of firm $B$ when the type of firm $B$ is $w \in I_{B}$. We note that $E\left(p_{A}\right)=E_{A}\left(p_{A}\right)$ and $E\left(p_{B}\right)=E_{B}\left(p_{B}\right)$. The indifferent consumer $x: I_{A} \times I_{B} \rightarrow(0, l)$ is given by

$$
x^{z, w}=\frac{p_{B}^{w}-p_{A}^{z}+t m\left(l+\Delta_{l}\right)}{2 t m} .
$$


The ex-post profit of the firms is the effective profit of the firms given a realization of the production costs for both firm. Hence, it is the main economic information for both firms. However, the incomplete information prevents the firms to have access to their ex-post profits except after the firms have already decided their price strategies. The ex-post profits $\pi_{A}^{E P}: I_{A} \times I_{B} \rightarrow \mathbb{R}_{0}^{+}$and $\pi_{B}^{E P}: I_{A} \times I_{B} \rightarrow \mathbb{R}_{0}^{+}$are given by

$$
\pi_{A}^{E P}(z, w)=\pi_{A}(z, w)=\left(p_{A}^{z}-c_{A}^{z}\right) x^{z, w}
$$

and

$$
\pi_{B}^{E P}(z, w)=\pi_{B}(z, w)=\left(p_{B}^{w}-c_{B}^{w}\right)\left(l-x^{z, w}\right) .
$$

The ex-ante profit of the firms is the expected profit of the firm that know their production cost but are uncertain about the production cost of the competitor firm. The ex-ante profits $\pi_{A}^{E A}: I_{A} \rightarrow \mathbb{R}_{0}^{+}$and $\pi_{B}^{E A}: I_{B} \rightarrow \mathbb{R}_{0}^{+}$are given by

$$
\pi_{A}^{E A}(z)=E_{B}\left(\pi_{A}^{E P}\right) \text { and } \pi_{B}^{E A}(w)=E_{A}\left(\pi_{B}^{E P}\right)
$$

We note that, the expected profit $E\left(\pi_{A}^{E P}\right)$ of firm $A$ is equal to $E_{A}\left(\pi_{A}^{E A}\right)$ and the expected profit $E\left(\pi_{B}^{E P}\right)$ of firm $B$ is equal to $E_{B}\left(\pi_{B}^{E A}\right)$.

The incomplete information forces the firms to have to choose their price strategies using their knowledge of their ex-ante profits, to which they have access, instead of the ex-post profits, to which they do not have access except after the price strategies are decided.

Definition 34.3. A price strategy $\left(p_{A}, p_{B}\right)$ for both firms is a local optimum price strategy if (i) for every $z \in I_{A}$ and for every small deviation of the price $p_{A}^{z}$ the ex-ante profit $\pi_{A}^{E A}(z)$ of firm $A$ decreases, and for every $w \in I_{B}$ and for every small deviation of the price $p_{B}^{w}$ the ex-ante profit $\pi_{B}^{E A}(w)$ of firm $B$ decreases (local optimum property); and (ii) for every $z \in I_{A}$ and $w \in I_{B}$ the indifferent consumer exists, i.e. $0<x^{z, w}<l$ (duopoly property).

We introduce the BUCL1 condition that has the crucial economical information that can be extracted from the exogenous variables. The BUCL1 condition allow us to know if there is, or not, a local optimum price strategy in the presence of uncertainty for the production costs of both firms.

Definition 34.4. The Hotelling model satisfies the bounded uncertain costs and location (BUCL1) condition 1, if

$$
\left|\Delta_{E}-3 \Delta_{C}+2 \Delta_{l} t m\right|<6 t m l \text {. }
$$

for all $z \in I_{A}$ and for all $w \in I_{B}$.

A strong restriction that the $B U C L 1$ condition imposes is that $\Delta_{C}$ converges to 0 when $m$ tends to 0 , i.e. when the differentiation in the localization tends to vanish. 
For $i \in\{A, B\}$, we define

$$
c_{i}^{m}=\min _{z \in I_{i}}\left\{c_{i}^{z}\right\} \text { and } c_{i}^{M}=\max _{z \in I_{i}}\left\{c_{i}^{z}\right\}
$$

Let

$$
\bar{\Delta}=\max _{i, j \in\{A, B\}}\left\{c_{i}^{M}-c_{j}^{m}\right\}
$$

Thus, the bounded uncertain costs and location BUCL1 is implied by the following stronger SBUCL1 condition.

Definition 34.5. The Hotelling model satisfies the bounded uncertain costs and location (SBUCL1) condition, if

$$
\bar{\Delta}<t l m \text {. }
$$

The following theorem is a key economical result in oligopoly theory. First, it tell us about the existence, or not, of a local optimum price strategy only by accessing a simple inequality in the exogenous variables and so available to both firms. Secondly, give us explicit and simple formulas that allow the firms to know the relevance of the exogenous variables in their price strategies and corresponding profits.

Theorem 34.1. There is a local optimum price strategy $\left(p_{A}, p_{B}\right)$ if and only if the BUCL1 condition holds. Under the BUCL1 condition, the expected prices of the local optimum price strategy are given by

$$
E\left(p_{A}\right)=t m\left(l+\frac{\Delta_{l}}{3}\right)+E\left(c_{A}\right)-\frac{\Delta_{E}}{3} ; E\left(p_{B}\right)=t m\left(l-\frac{\Delta_{l}}{3}\right)+E\left(c_{B}\right)+\frac{\Delta_{E}}{3} .
$$

Furthermore, the local optimum price strategy $\left(p_{A}, p_{B}\right)$ is unique and it is given by

$$
p_{A}^{z}=E\left(p_{A}\right)+\frac{\Delta_{A}}{2} ; \quad p_{B}^{w}=E\left(p_{B}\right)+\frac{\Delta_{B}}{2} .
$$

We observe that the differences between the expected prices of both firms has a very useful and clear economical interpretation in terms of the localization and expected cost deviations.

$$
E\left(p_{A}\right)-E\left(p_{B}\right)=\frac{2 t m \Delta_{l}+\Delta_{E}}{3} .
$$

Furthermore, for different production costs, the differences between the optimal prices of a firm are proportional to the differences of the production costs 


$$
p_{A}^{z_{1}}-p_{A}^{z_{2}}=\frac{c_{A}^{z_{1}}-c_{A}^{z_{2}}}{2}
$$

and

$$
p_{B}^{w_{1}}-p_{B}^{w_{2}}=\frac{c_{B}^{w_{1}}-c_{B}^{w_{2}}}{2}
$$

for all $z_{1}, z_{2} \in I_{A}$ and $w_{1}, w_{2} \in I_{B}$. Hence, half of the production costs value is incorporated in the price.

The following equation give us the information of the market size of both firms by giving the explicit localization of the indifferent consumer $x^{z, w}$ with respect to the local optimum price strategy

$$
x^{z, w}=\frac{1}{2}\left(l+\frac{\Delta_{l}}{3}\right)+\frac{\Delta_{E}-3 \Delta_{C}}{12 t m} .
$$

The ex-ante profit of firm $A$ is

$$
\pi_{A}^{E A}(z)=\frac{\left(2 t m\left(3 l+\Delta_{l}\right)-3 \Delta_{A}-2 \Delta_{E}\right)^{2}}{72 t m} .
$$

Similarly, the ex-ante profit of firm $B$ is

$$
\pi_{B}^{E A}(w)=\frac{\left(2 t m\left(3 l-\Delta_{l}\right)-3 \Delta_{B}+2 \Delta_{E}\right)^{2}}{72 t m} .
$$

\subsection{Bayesian-Nash Equilibrium}

We note that, if a Bayesian-Nash price equilibrium satisfies the duopoly property then it is a local optimum price strategy. However, a local optimum price strategy is only a local strategic maximum. Hence, the local optimum price strategy to be a Bayesian-Nash equilibrium must also be global strategic maximum. In this section, we are going to show that this is the case.

Following D'Aspremont et al. [4], we note that the profits of the two firms, valued at local optimum price strategy are globally optimal if they are at least as great as the payoffs that firms would earn by undercutting the rivals's price and supplying the whole market for all admissible subsets of types $I_{A}$ and $I_{B}$.

Let $\left(p_{A}, p_{B}\right)$ be the local optimum price strategy. Given the type $w_{0}$ of firm $B$, firm $A$ may gain the whole market, undercutting its rival by setting

$$
p_{A}^{M}\left(w_{0}\right)=p_{B}^{w_{0}}-t m\left(l-\Delta_{l}\right)
$$


Hence, by $B U C L 1$ condition $p_{A}^{M}\left(w_{0}\right) \leqslant p_{A}^{z}$ for all $z \in I_{A}$. We observe that if firm $A$ chooses the price $p_{A}^{M}\left(w_{0}\right)$ then by equalities (34.9) and (34.10) the whole market belongs to Firm $A$ for all types $w$ of firm $B$ with $c^{w} \geqslant c^{w_{0}}$. Let

$$
x\left(w ; w_{0}\right)=\min \left\{l, \frac{p_{B}^{w}-p_{A}^{M}\left(w_{0}\right)}{2 t m}+\frac{l+\Delta_{l}}{2}\right\} .
$$

Thus, the expected profit with respect to the price $p_{A}^{M}\left(w_{0}\right)$ for firm $A$ is

$$
\pi_{A}^{E A, M}\left(w_{0}\right)=\int_{I_{B}}\left(p_{A}^{M}\left(w_{0}\right)-c_{A}^{z}\right) x\left(w ; w_{0}\right) \mathrm{d} q_{B}(w) .
$$

Let $w_{M} \in I_{B}$ such that $c^{w_{M}}=c_{B}^{M}$. Since $c^{w_{M}} \geqslant c_{B}^{w_{0}}$ for every $w_{0} \in I_{B}$, we obtain

$$
\pi_{A}^{E A, M}\left(w_{0}\right) \leqslant\left(p_{A}^{M}\left(w_{0}\right)-c_{A}^{z}\right) l \leqslant\left(p_{A}^{M}\left(w_{M}\right)-c_{A}^{z}\right) l
$$

Given the type $z_{0}$ of firm $A$, firm $B$ may gain the whole market, undercutting its rival by setting

$$
p_{B}^{M}\left(z_{0}\right)=p_{A}^{z_{0}}-t m\left(l+\Delta_{l}\right)
$$

Hence, by $B U C L 1$ condition $p_{B}^{M}\left(z_{0}\right) \leqslant p_{B}^{w}$ for all $w \in I_{B}$. We observe that if firm $B$ chooses the price $p_{B}^{M}\left(z_{0}\right)$ then by equalities (34.9) and (34.10) the whole market belongs to Firm $B$ for all types $z$ of firm $A$ with $c^{z} \geqslant c^{z_{0}}$. Let

$$
x\left(z ; z_{0}\right)=\max \left\{0, \frac{p_{B}^{M}\left(z_{0}\right)-p_{A}^{z}}{2 t m}+\frac{l+\Delta_{l}}{2}\right\} .
$$

Thus, the expected profit with respect to the price $p_{B}^{M}\left(z_{0}\right)$ of firm $B$ is

$$
\pi_{B}^{E A, M}\left(z_{0}\right)=\int_{I_{A}}\left(p_{B}^{M}\left(z_{0}\right)-c_{B}^{w}\right)\left(l-x\left(z ; z_{0}\right)\right) \mathrm{d} q_{A}(z) .
$$

Let $z_{M} \in I_{A}$ such that $c_{A}^{z_{M}}=c_{A}^{M}$. Since $c^{z_{M}} \geqslant c^{z_{0}}$ for every $z_{0} \in I_{A}$, we obtain

$$
\pi_{B}^{E A, M}\left(z_{0}\right) \leqslant\left(p_{B}^{M}\left(z_{0}\right)-c_{B}^{w}\right) l \leqslant\left(p_{B}^{M}\left(z_{M}\right)-c_{B}^{w}\right) l .
$$

Remark 34.1. Under the BUCL1 condition, the strategic equilibrium $\left(p_{A}, p_{B}\right)$ is the unique pure Bayesian Nash equilibrium with the duopoly property if for every $z \in I_{A}$ and every $w \in I_{B}$,

$$
\pi_{A}^{E A, M}(w) \leqslant \pi_{A}^{E A}(z) \quad \text { and } \quad \pi_{B}^{E A, M}(z) \leqslant \pi_{B}^{E A}(w) .
$$


Definition 34.6. The Hotelling model satisfies the bounded uncertain costs and location (BUCL2) condition 2, if

$$
\begin{gathered}
\Delta_{E}+3\left(c_{A}^{M}+c_{B}^{M}-2 c_{A}^{m}\right)+\frac{\Delta_{l}\left(3 c_{A}^{M}-E\left(c_{A}\right)-2 E\left(c_{B}\right)\right)}{3 l} \leqslant \\
\leqslant \frac{\left(3 l-\Delta_{l}\right)^{2} t m}{3 l}+\frac{\left(3 c_{A}^{M}-E\left(c_{A}\right)-2 E\left(c_{B}\right)\right)^{2}}{12 t m l}
\end{gathered}
$$

and

$$
\begin{aligned}
-\Delta_{E}+ & 3\left(c_{A}^{M}+c_{B}^{M}-2 c_{B}^{m}\right)-\frac{\Delta_{l}\left(3 c_{B}^{M}-E\left(c_{B}\right)-2 E\left(c_{A}\right)\right)}{3 l} \leqslant \\
& \leqslant \frac{\left(3 l+\Delta_{l}\right)^{2} t m}{3 l}+\frac{\left(3 c_{B}^{M}-E\left(c_{B}\right)-2 E\left(c_{A}\right)\right)^{2}}{12 t m l} .
\end{aligned}
$$

Thus, the bounded uncertain costs condition BUCL2 is implied by the following stronger SBUCL2 condition.

Definition 34.7. The Hotelling model satisfies the strong bounded uncertain costs and location (SBUCL2) condition 2, if

$$
6 \bar{\Delta}<l t m
$$

We observe that the SBUCL2 condition implies $S B U C L 1$ condition and so implies the $B U C L 1$ condition.

Theorem 34.2. If the Hotelling model satisfies the BUCL1 and BUCL2 conditions the local optimum price strategy $\left(p_{A}, p_{B}\right)$ is a Bayesian Nash equilibrium.

Corollary 34.1. If the Hotelling model satisfies SBUCL2 condition the local optimum price strategy $\left(p_{A}, p_{B}\right)$ is a Bayesian Nash equilibrium.

\subsection{Optimum Localization Equilibrium Under Incomplete Information}

We note that from (34.10) and (34.11), we can write the profit of firm $A$ as

$$
\pi_{A}^{E A}(z)=\frac{\left(p_{A}^{z}-c_{A}\right)^{2}}{2 t(l-a-b)} .
$$

Since

$$
\frac{\partial p_{A}^{z}}{\partial a}=-\frac{2}{3} t(l+a)
$$


we have

$$
\frac{\partial \pi_{A}^{E A}}{\partial a}=\frac{p_{A}-c_{A}}{12 t(l-a-b)^{2}}\left(-2 t(l-a-b)(l+3 a+b)-3 \Delta_{A}-2 \Delta_{E}\right) .
$$

Similarly, we obtain that

$$
\frac{\partial \pi_{B}^{E A}}{\partial b}=\frac{p_{B}-c_{B}}{12 t(l-a-b)^{2}}\left(-2 t(l-a-b)(l+3 b+a)-3 \Delta_{B}+2 \Delta_{E}\right) .
$$

Therefore, the maximal differentiation $(a, b)=(0,0)$ is a local optimum strategy if and only if

$$
\frac{\partial \pi_{A}^{E A}}{\partial a}(0,0)=-\frac{p_{A}-c_{A}}{12 t l^{2}}\left(2 t l^{2}+3 \Delta_{A}+2 \Delta_{E}\right)<0
$$

and

$$
\frac{\partial \pi_{B}^{E A}}{\partial b}(0,0)=-\frac{p_{B}-c_{B}}{12 t l^{2}}\left(2 t l^{2}+3 \Delta_{B}-2 \Delta_{E}\right)<0
$$

Since

$$
\frac{p_{A}-c_{A}}{6 t l^{2}}>0 \text { and } \quad \frac{p_{B}-c_{B}}{6 t l^{2}}>0
$$

the maximal differentiation $(a, b)=(0,0)$ is a local optimum strategy if and only if the following condition holds.

Definition 34.8. The Hotelling model satisfies the bounded uncertain costs and location (BUCL3) condition, if

$$
2 t l^{2}+3 \Delta_{A}+2 \Delta_{E}>0
$$

for all $z \in I_{A}$ and

$$
2 t l^{2}+3 \Delta_{B}-2 \Delta_{E}>0 .
$$

for all $w \in I_{B}$.

\subsection{Conclusion}

We proved that there is a local optimum price strategy with the duopoly property if and only if the bounded uncertain costs and location BUCL1 condition holds. The explicit formulas of the local optimum price strategy determine prices for both 
firms that are affine with respect to the expected costs of both firms and to its own costs. Under the BUCL1 and BUCL2 conditions, we proved that the local optimum price strategy is a Bayesian-Nash price strategy. Assuming that the firms choose the Bayesian-Nash price strategy, under the BUCL3 condition, we proved that the maximal differentiation is a local optimum for the localization strategy of both firms.

Acknowledgements We are thankful to the anonymous referees for their suggestions. We acknowledge the financial support of LIAAD-INESC TEC through 'Strategic Project-LA 142013-2014' with reference PEst-C/EEI/LA0014/2013, USP-UP project, IJUP, Faculty of Sciences, University of Porto, Calouste Gulbenkian Foundation, FEDER, POCI 2010 and COMPETE Programmes and Fundação para a Ciência e a Tecnologia (FCT) through Project "Dynamics and Applications", with reference PTDC/MAT/121107/2010. Telmo Parreira thanks FCT, for the PhD scholarship SFRH/BD/33762/2009.

\section{References}

1. Biscaia, R., Sarmento, P.: Spatial Competition and Firms' Location Decisions under Cost Uncertainty. FEP Working Papers No. 445 (2012)

2. Boyer, M., Laffont, J., Mahenc, P., Moreaux, M.: Location Distortions under Incomplete information. Reg. Sci. Urban Econ. 24(4), 409-440 (1994)

3. Boyer, M., Mahenc, P., Moreaux, M.: Asymmetric information and product differentiation. Reg. Sci. Urban Econ. 33(1), 93-113 (2003)

4. D'Aspremont, C., Gabszewicz, J., Thisse, J.-F.: On hotelling's "stability in competition". Econometrica 47(5), 1145-1150 (1979)

5. Ferreira, F.A., Pinto, A.A.: Uncertainty on a Bertrand duopoly with product differentiation. In: Machado, J.A., Silva, M.F., Barbosa, R.S., Figueiredo, L.B. (eds.) Nonlinear Science and Complexity, pp. 389-395. Springer, Netherlands (2011)

6. Ferreira, F., Ferreira, F.A., Ferreira, M., Pinto, A.A.: Flexibility in a Stackelberg leadership with differentiated goods. Optimization (accepted)

7. Ferreira, F., Ferreira, F.A., Pinto, A.A.: Bayesian price leadership. In: Tas, K., et al. (eds.) Mathematical Methods in Engineering, pp. 371-379. Springer, Netherlands (2007)

8. Ferreira, F.A., Ferreira, F., Pinto, A.A.: Unknown costs in a duopoly with differentiated products. In: Tas, K., et al. (eds.) Mathematical Methods in Engineering, pp. 359-369. Springer, Netherlands (2007)

9. Ferreira, F., Ferreira, F.A., Pinto, A.A.: Flexibility in stackelberg leadership. In: Machado, J.A., Patkai, B., Rudas, I.J. (eds.) Intelligent Engineering Systems and Computational Cybernetics, pp. 399-405. Springer, Netherlands (2009)

10. Ferreira, F., Ferreira, F.A., Pinto, A.A.: Price-setting dynamical duopoly with incomplete information. In: Machado, J.A., Silva, M.F., Barbosa, R.S., Figueiredo, L.B. (eds.) Nonlinear Science and Complexity, pp. 397-403. Springer, Netherlands (2011)

11. Ferreira, M., Figueiredo, I.P., Oliveira, B.M.P.M., Pinto, A.A.: Strategic optimization in R\&D Investment. Optim. J. Math. Program. Oper. Res. 61(8), 1013-1023 (2012)

12. Gibbons, R.: A Primer in Game Theory. Prentice Hall (1992)

13. Graitson, D.: Spatial competition à la Hotelling: a selective survey. J. Ind. Econ. 31, 11-25 (1982)

14. Hotelling, H.: Stability in competition. Econ. J. 39, 41-57 (1929)

15. Lederer, P., Hurter, A.: Competition of Firms: discriminatory pricing and location. Econometrica 54(3), 623-640 (1986) 
16. Osborne, M.J., Pitchick, C.: Equilibrium in Hotelling's model of spatial competition. Econometrica 55(4), 911-922 (1987)

17. Pinto, A.A., Parreira, T.: A hotelling-type network. In: Peixoto, M., Pinto, A.A., Rand, D. (eds.) Dynamics, Games and Science I. Springer Proceedings in Mathematics, vol. 1, pp. 709-720. Springer, Berlin (2011)

18. Pinto, A.A., Parreira, T.: Complete versus incomplete information in the Hotelling model. In: Pinto, A.A., Zilberman, D. (eds.) Modeling, Dynamics, Optimization and Bioeconomy. Springer Proceedings in Mathematics and Statistics Series. Springer, Heidelberg (2014)

19. Pinto, A.A., Parreira, T.: Optimal localization of firms in Hotelling networks. In: Pinto, A.A., Zilberman, D. (eds.) Modeling, Dynamics, Optimization and Bioeconomy. Springer Proceedings in Mathematics and Statistics Series. Springer, Heidelberg (2014)

20. Pinto, A.A., Parreira, T.: Price competition in the Hotelling model with uncertainty on costs. Optim. J. Math. Program. Oper. Res. (accepted)

21. Pinto, A.A., Parreira, T.: Localization and prices in the quadratic Hotelling model with uncertainty (submited)

22. Pinto, A.A., Ferreira, F.A., Ferreira, M., Oliveira, B.M.P.M.: Cournot duopoly with competition in the R\&D expenditures. In: Proceedings of Symposia in Pure Mathematics, vol. 7. Wiley-VCH Verlag, Weinheim, 2007

23. Pinto, A.A., Oliveira, B.M.P.M., Ferreira, F.A., Ferreira, F.: Stochasticity favoring the effects of the R\&D strategies of the firms. In: Machado, J.A., Patkai, B., Rudas, I.J. (eds.) Intelligent Engineering Systems and Computational Cybernetics, pp. 415-423. Springer, Netherlands (2009)

24. Salop, S.: Monopolistic competition with outside goods. Bell J. Econ. 10, 141-156 (1979)

25. Tabuchi, T., Thisse, J.F.: Asymmetric equilibria in spatial competition. Int. J. Econ. Theory 13(2), 213-227 (1995)

26. Ziss, S.: Entry deterrence, cost advantage and horizontal product differentiation. Reg. Sci. Urban Econ. 23, 523-543 (1993) 